\title{
Evolutionary implications of the new triple- $\alpha$ nuclear reaction rate for low mass stars (Research Note)
}

\author{
A. Dotter ${ }^{1}$ and B. Paxton ${ }^{2}$ \\ 1 Department of Physics and Astronomy, University of Victoria, Victoria, BC, V8P 5C2 Canada \\ e-mail: dotter@uvic.ca \\ 2 Kavli Institute for Theoretical Physics, University of California, Santa Barbara, Santa Barbara, CA 93106 USA \\ e-mail: paxton@kitp.ucsb.edu
}

Received 27 July 2009 / Accepted 17 September 2009

ABSTRACT

\begin{abstract}
Context. Ogata et al. (2009, Progr. Theor. Phys., 122, 1055) presented a theoretical determination of the ${ }^{4} \mathrm{He}(\alpha \alpha, \gamma)^{12} \mathrm{C}$, or triple- $\alpha$, nuclear reaction rate. Their rate differs from the NACRE rate by many orders of magnitude at temperatures relevant for low mass stars.

Aims. We explore the evolutionary implications of adopting the OKK triple- $\alpha$ reaction rate in low mass stars and compare the results with those obtained using the NACRE rate.

Methods. The triple- $\alpha$ reaction rates are compared by following the evolution of stellar models at 1 and $1.5 M_{\odot}$ with $Z=0.0002$ and $Z=0.02$.

Results. Results show that the OKK rate has severe consequences for the late stages of stellar evolution in low mass stars. Most notable is the shortening-or disappearance-of the red giant phase.

Conclusions. The OKK triple- $\alpha$ reaction rate is incompatible with observations of extended red giant branches and He burning stars in old stellar systems.
\end{abstract}

Key words. nuclear reactions, nucleosynthesis, abundances - stars: evolution - stars: Hertsprung-Russell (HR) and C-M diagrams

\section{Introduction}

Ogata et al. (2009, hereafter OKK) introduced a new determination of the triple- $\alpha$ nuclear reaction rate based on a direct solution of the Schrödinger equation. The authors provided a tabulation of the triple- $\alpha$ rate for temperatures from $10^{7}$ to $10^{9} \mathrm{~K}$ and also the ratio of their rate to the NACRE (Angulo et al. 1999) rate. The OKK rate is a factor $10^{26}$ greater than the NACRE rate at $10^{7} \mathrm{~K}, 10^{6}$ times greater at $10^{8} \mathrm{~K}$, and reaches equality at $25 \times 10^{8} \mathrm{~K}$ and above. It is important to note that OKK normalized their rate to the NACRE result at $10^{9} \mathrm{~K}$. For comparison, the previous update to the triple- $\alpha$ reaction rate was from Fynbo et al. (2005). Between $10^{7}$ and $10^{9} \mathrm{~K}$, the rate determined by Fynbo et al. differed from the NACRE rate by a factor of $\sim 3$ at most. The previous standard rate, that of Caughlan \& Fowler (1988), was generally lower than the NACRE rate but never by more than a factor of $\sim 10$.

The stellar evolutionary implications of uncertainties in the triple- $\alpha$ reaction have recently been considered by Weiss et al. (2005), who found that the difference between the Fynbo et al. (2005) rate and the NACRE rate made very little difference in the evolution of low- and intermediate-mass stars from $Z=0$ to solar. Herwig et al. (2006) explored the implications of changing the triple- $\alpha$ rate (and two other rates) within the uncertainties provided by the NACRE collaboration in the evolution and stellar yield from a $2 M_{\odot}, Z=0.01$ model evolved through the thermally pulsing AGB. Their calculations revealed that, within the uncertainties, the amount of material dredged up during varied by a factor of $\sim 2$ and the yield of ${ }^{12} \mathrm{C}$ varied by a factor of $\sim 3$.
These are significant differences brought about by uncertainties in the triple- $\alpha$ reaction rate of $\sim 10-15 \%$.

By contrast the tremendous difference at low temperatures predicted by the OKK rate is certain to have dramatic implications for stellar evolution calculations and it was with this in mind that we undertook the following investigation. The purpose of this research note is simple: to investigate the evolutionary implications of the new triple- $\alpha$ rate and compare results obtained from the new rate with results based on the NACRE rate.

\section{Stellar evolution model comparison}

Stellar evolution calculations were carried out using two codes: DSEP (Dotter et al. 2007) and MESA (an open source stellar evolution code developed by B. Paxton and collaborators; http://mesa.sourceforge.net/). The input physics employed by DSEP and MESA are similar for the models presented here. Both codes use a combination of OPAL (Iglesias \& Rogers 1996) and Ferguson et al. (2005) radiative opacities. For simplicity, the nuclear reaction networks in both codes were set to use the NACRE rates for all relevant $\mathrm{H}$ - and Heburning reactions, except that either the NACRE or OKK rate was used for the triple- $\alpha$ reaction. The OKK rate was implemented by interpolation within a table. The rate table, which included additional points at lower temperatures than in the published table, was provided to VandenBerg by Ogata via private communication. The table was interpolated using the 


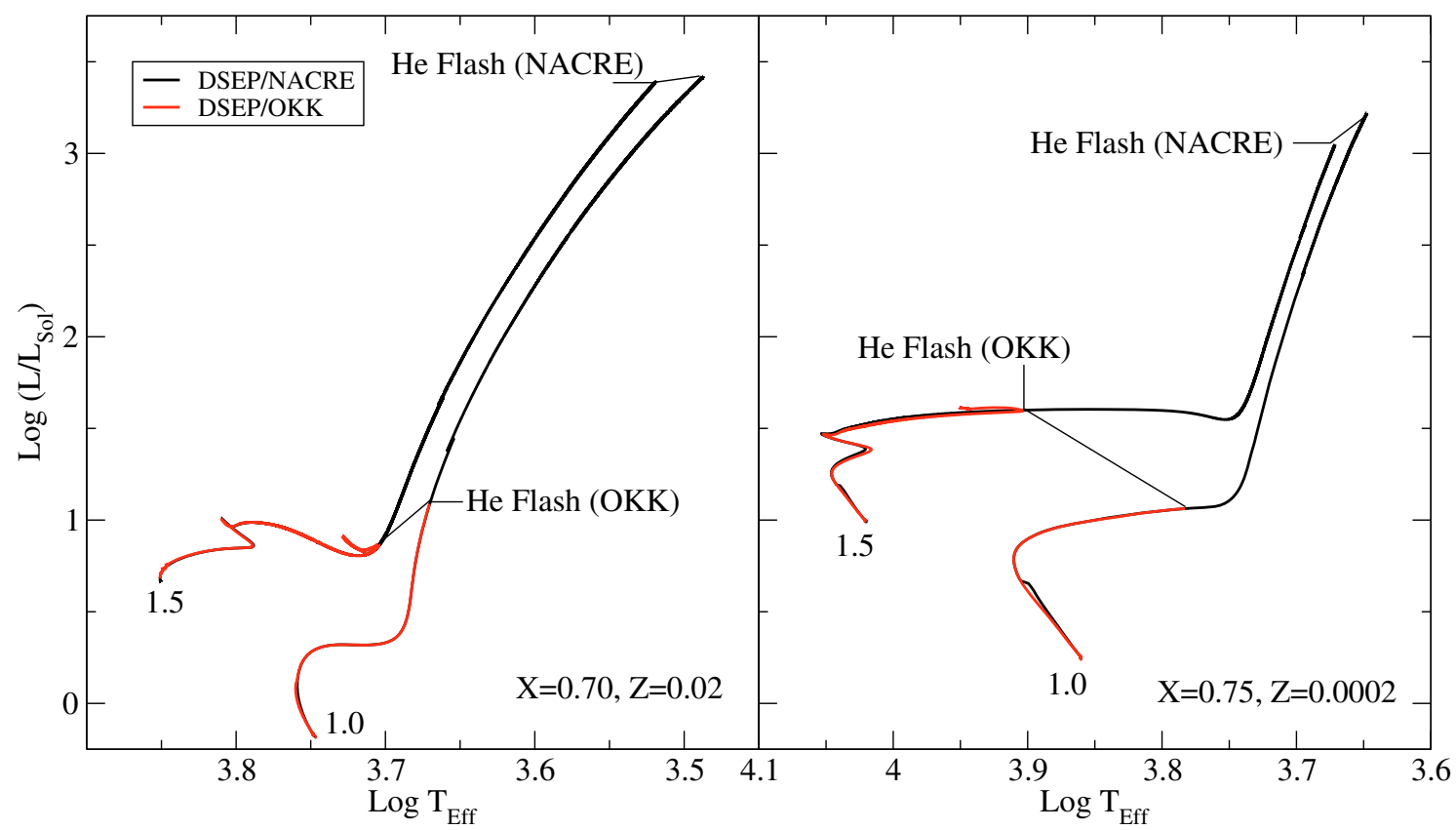

Fig. 1. DSEP models in the H-R diagram for $X=0.7, Z=0.02$ (left panel) and $X=0.75, Z=0.0002$ (right panel). Evolutionary tracks for 1 and $1.5 M_{\odot}$ are shown in both panels.

monotonicity-preserving routine developed by Steffen (1990) ${ }^{1}$. For the equation of state, DSEP uses the FreeEOS code (http://freeeos.sourceforge.net) while MESA uses a combination of OPAL (Rogers \& Nayfonov 2002) and SCVH tables (Saumon et al. 1995) along with the HELM EOS (Timmes $\&$ Swesty 2000) for temperatures and densities corresponding to He-burning and beyond. DSEP models use a mixing length parameter of $\alpha_{\mathrm{MLT}}=1.94$ and MESA models use $\alpha_{\mathrm{MLT}}=2.14$. The DSEP models include gravitational settling of $\mathrm{He}$ and metals while the MESA models do not.

Stellar models were computed at 1 and $1.5 M_{\odot}$ with initial $X=0.70, Z=0.02$ using both codes. DSEP also produced models at the same masses with $X=0.75, Z=0.0002$. DSEP followed the evolution from the fully-convective pre-main sequence phase until the onset of the core He flash. MESA followed the evolution from the ZAMS through the core He flash and core He burning phases. The pre-main sequence evolution is not affected by the triple- $\alpha$ process and is not shown in the following figures for clarity.

Figure 1 shows the H-R diagram with DSEP models at 1 and $1.5 M_{\odot}$ : the left panel includes tracks at $X=0.7, Z=0.02$ and the right panel shows tracks at $X=0.75, Z=0.0002$. Both panels clearly demonstrate that the DSEP/NACRE models experience an extended red giant phase in all cases. Conversely, the DSEP/OKK models show little or no red giant phase, with the $Z=0.0002$ models transitioning to core He-burning while still in the subgiant phase. The OKK rate also causes a very modest increase in surface temperature during the main sequence but the effect is small and difficult to see in the figure.

Figure 2 shows, in the left panel, the H-R diagram of the $1 M_{\odot}, Z=0.02$ model evolved through the core He flash and core He-burning phase by the MESA code. The MESA/OKK model has a core He-burning phase that occurs at $\sim 3-4 L_{\odot}$ : about an order of magnitude fainter than the core He-burning phase experienced by MESA/NACRE model. The right panel of

\footnotetext{
1 A FORTRAN implementation of the interpolation routine is provided as a part of the MESA package.
}

Fig. 2 shows the late-time evolution of the same models. Because the MESA/OKK model undergoes core He-burning at such low luminosity, it is able to continue for about $1 \mathrm{Gyr}$ at more or less constant luminosity. The MESA/NACRE model experiences core He-burning for $\sim 100 \mathrm{Myr}$ at roughly ten times the luminosity of the MESA/OKK model.

Figure 3 shows the central temperature-density diagrams for the $1 M_{\odot}, Z=0.02$ models with DSEP on the left and MESA on the right. Models evolved with both codes trace out identical paths in this plane until the DSEP models terminate at the onset of the core He flash. The MESA tracks show the evolution through the core He flash and core He-burning phases. The figure demonstrates that the OKK rate causes the core He flash to occur at a much lower temperature ( $\sim 30$ million $\mathrm{K}$ vs. $\sim 80$ million $\mathrm{K}$ ) and an order of magnitude lower density than the NACRE rate.

\section{Conclusions}

Comparisons of stellar evolution models computed with NACRE and OKK triple- $\alpha$ reaction rates were presented. The OKK rates cause models of low mass stars to have either a shortened red giant phase or to bypass the red giant phase altogether. The OKK rate also causes core He-burning to take place at a factor of ten lower luminosity-and for a factor ten longer duration-than the NACRE rate.

Given the excellent agreement between stellar evolution models and observations of the RGB (Salaris et al. 2002) when the models employ the NACRE (or relatively similar) triple- $\alpha$ reaction rates, such as Caughlan \& Fowler (1988) or Fynbo et al. (2005), it is difficult to envision how such a dramatic change as that proposed by OKK could lead to improved agreement with observations. Indeed, the triple- $\alpha$ reaction rate determined by OKK is in stark disagreement with fundamental observational evidence of stellar evolution, namely: (1) the existence of extended red giant branches in old stellar systems; (2) the location of core He-burning stars in the H-R or color-magnitude diagram; and (3) the lifetime ratio of the core He-burning phase to the red giant phase as manifested by the number ratio of horizontal 


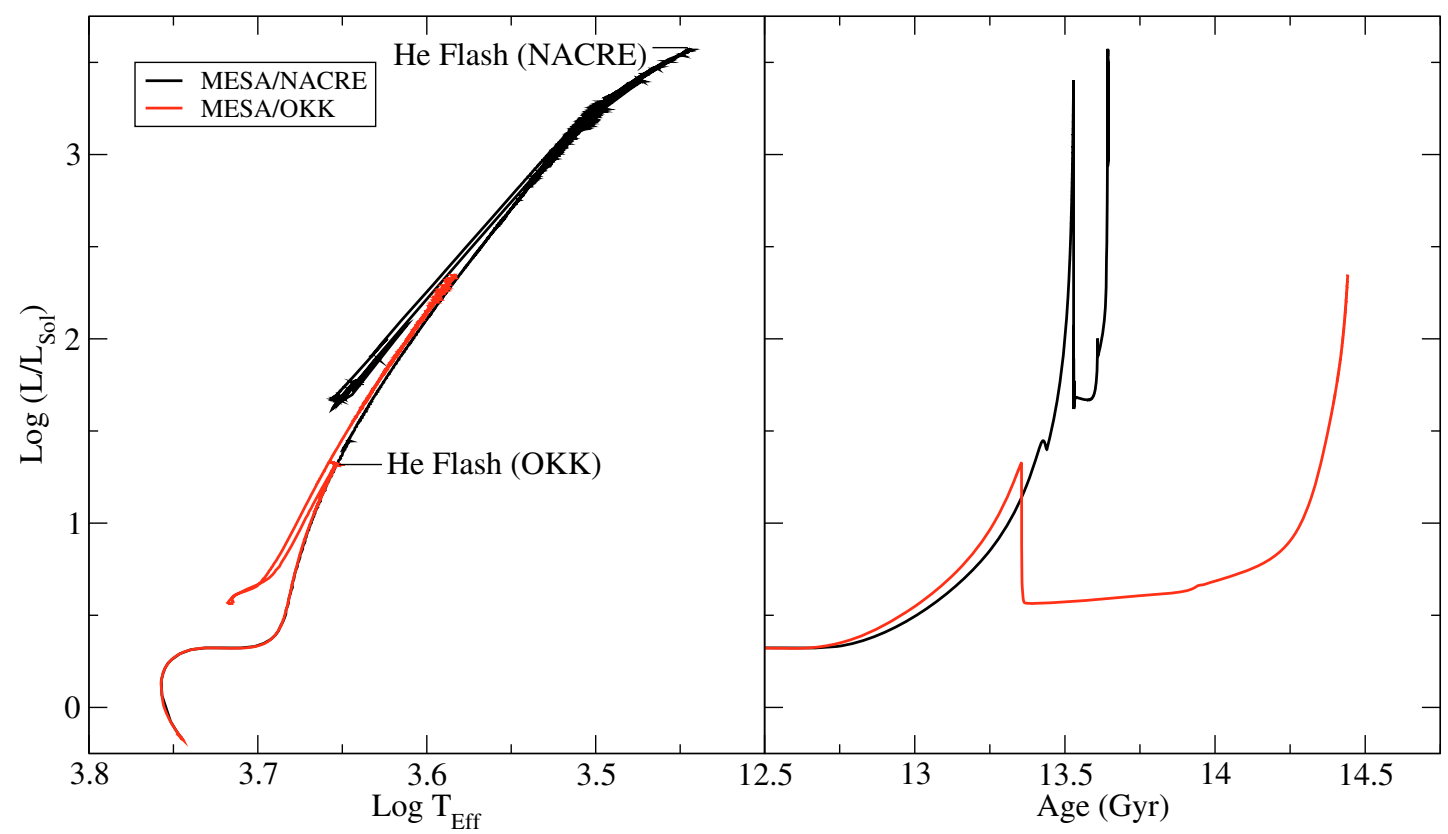

Fig. 2. The H-R diagram (left panel) and age-luminosity diagram (right panel) from the $1 M_{\odot}, Z=0.02$ MESA model. MESA is able to evolve the models through the core He flash and both tracks show core He-burning phases in the H-R diagram. The right panel demonstrates the prolonged core He-burning phase that results from adopting the OKK triple- $\alpha$ rate.

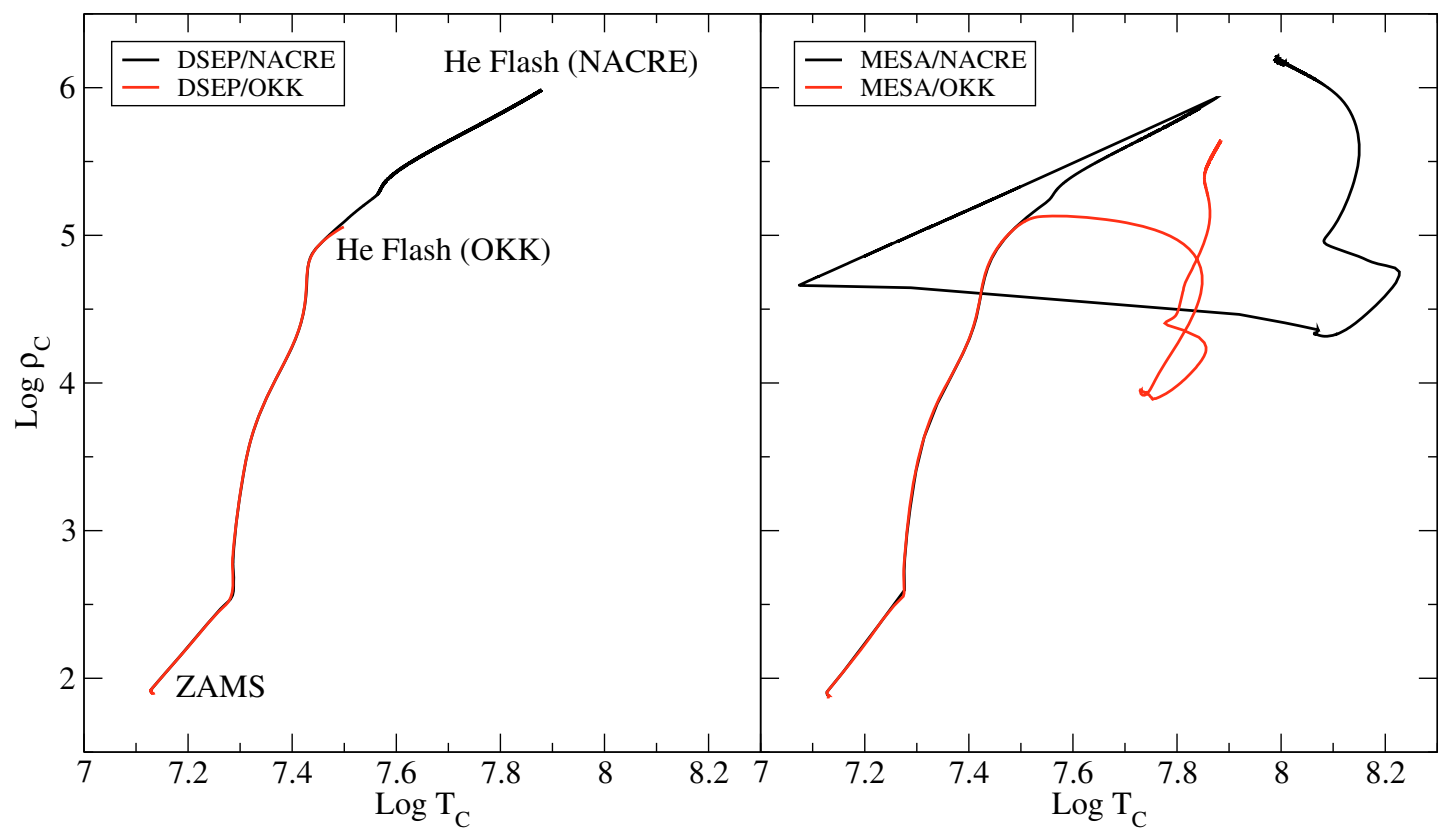

Fig. 3. Central temperature-density diagram for $1 M_{\odot}$ models at $Z=0.02$. The left panel shows the DSEP models and labels the location of the ZAMS and core He flash for both models. The right panel shows MESA models, which also show the core He-burning phase of evolution.

branch stars to RGB stars, also known as the R parameter (e.g. Cassisi et al. 2003; Salaris et al. 2005).

Acknowledgements. A.D. was supported by a CITA National Fellowship and by an NSERC grant to D. VandenBerg. B.P. was supported by the National Science Foundation under grants PHY 05-51164 and AST 07-07633.

\section{References}

Angulo, C., Arnould, M., Rayet, M., et al. 1999, Nucl. Phys. A, 656, 3 Cassisi, S., Salaris, M., \& Irwin, A. W. 2003, ApJ, 588, 862

Caughlan, G. A., \& Fowlwer, W. A. 1988, At. Data Nucl. Data Tables, 40, 283 Dotter, A., Chaboyer, B., Jevremovic, D., et al. 2007, AJ, 134, 376
Ferguson, J. W., Alexander, D. R., Allard, F., et al. 2005, ApJ, 623, 585 Fynbo, H. O. U., Diget, C. A., Bergmann, U. C., et al. 2005, Nature, 433, 136 Herwig, F., Austin, S. M., \& Lattanzio, J. C. 2006, Phys. Rev. C, 73, 025802 Iglesias, C. A., \& Rogers, F. J. 1996, ApJ, 464, 943

Ogata, K., Kan, M., \& Kamimura, M. 2009, Progr. Theor. Phys., 122, 1055 (OKK)

Rogers, F., \& Nayfonov, A. 2002, ApJ, 576, 1064

Salaris, M., Cassisi, S., \& Weiss, A. 2002, PASP, 114, 375

Salaris, M., Riello, M., Cassisi, S., \& Piotto, G. 2004, A\&A, 420, 911

Saumon, D., Chabrier, G., \& van Horn, M. H. 1995, ApJS, 99, 713S

Steffen, M. 1990, A\&A, 239, 443

Timmes, F. X., \& Swesty, F. D. 2000, ApJS, 126, 501

Weiss, A., Serenelli, A., Kitsikis, A., Schlattl, H., \& Christensen-Dalsgaard, J. 2005, A\&A, 441, 1129 